\title{
Laser contrast and other key parameters enhancing the laser conversion efficiency in ion acceleration regime
}

\author{
Lorenzo Torrisi \\ Dipartimento di Scienze Fisiche, MIFT, Università di Messina, \\ V.le F.S. D’Alcontres 31, 98166 S. Agata, Messina, Italy
}

\begin{abstract}
Measurements of ion acceleration in plasma produced by $f s$ lasers at intensity of the order of $10^{18} \mathrm{~W} / \mathrm{cm}^{2}$ have been performed in different European laboratories. The forward emission in targetnormal-sheath-acceleration (TNSA) regime indicated that the maximum energy is a function of the laser parameters, of the irradiation conditions and of the target properties.In particular the laser intensity and contrast play an important role to maximize the ion acceleration enhancing the conversion efficiency. Also the use of suitable prepulses, focal distances and polarized laser light has important roles. Finally the target composition, surface, geometry and multilayered structure, permit to enhance the electric field driving the forward ion acceleration.Experimental measurements will be reported and discussed.
\end{abstract}

\section{Introduction}

More and more progresses are achieved in the field of the ion acceleration using high intensity pulsed lasers, thank to many experiments and results obtained using different laser parameters. The high intensity lasers using $f_{s}$ pulse duration are accelerating ions above $1 \mathrm{MeV}$ per charge state and, by optimizing some important parameters of the used laser, irradiation conditions and target properties, recently are reaching values higher than $10 \mathrm{MeV}$ per charge state $[1,2]$.

The main mechanism used to accelerate ions at high kinetic energy is the target normal sheath acceleration (TNSA) occurring at the rear surface in forward direction [3]. The dominant accelerated ion species are protons, due to their high charge-to-mass ratio, which are always present as taking part of the target composition or as contaminant layers at the surfaces and interfaces.

The highest electron and ion density at the target rear surface, and consequently highest acceleration field obtained, is expected for ultrathin targets of a conducting material. The electric field of ion acceleration depends on the following parameters:

$$
E=\sqrt{\frac{k T_{e} n_{e}}{\varepsilon_{0}}}
$$

where $k T_{e}$ and $n_{e}$ represent the plasma electron temperature and density, respectively, and $\varepsilon_{0}$ is the vacuum permittivity [4].

Generally, as the target thickness is decreased, the transverse spreading of the relativistic electrons inside the target is reduced, resulting in a higher rear surface charge density and consequently in an higher ion acceleration and directionality along the normal to the target surface. For very high intensities and ultrathin targets, relativistic transparency allows part of the laser pulse to be transmitted through the target and contribute to increase the electron heating.

On the other hand, the thinning of the thickness of the target produces an increase in energy of the ions but also to a reduction of their yield, which therefore could decrease to such values as to be practically unusable.

One of the most severe limiting factors in laser-plasma ion acceleration is the presence of a laser pedestal of amplified spontaneous emission (ASE) or laser prepulses to the main pulse, which affects the laser-solid and laserplasma interaction [5]. A high pedestal, in facts, may destroy the solid target prior to the arrival of the main laser pulse, thus deteriorating the conditions to have better TNSA regime. From this point of view thin foils can be destroyed also by relatively low energetic pedestals with respect to thick foils, thus TNSA conditions and ion energy maximization need of a compromise between pedestal and main pulse intensities to obtain the best acceleration conditions.

The laser contrast, in terms of ratio between the intensity of the main pulse to that of the pedestal or prepulse, in fact, plays a fundamental role in the forward TNSA ion acceleration mechanism and the pedestal must be controlled accurately in duration and in intensity before to be applied to a given target irradiation.

I some cases the laser prepulse or the use of a laser focal positioning in front of the target may produce a preplasma in front of the solid target very useful to induce self-focusing effect [6], consisting in the increment of the refractive index in which the laser light may be further focalized up to spot dimensions comparable with the laser wavelength, so that the intensity increases, the

Corresponding author: lorenzo.torrisi@unime.it 
accelerated electron energy also and consequently the final ion acceleration enhanced.

However the key parameters to enhance the particle acceleration are many and some of them concern the target composition and structure, that must be employed to improve the TNSA mechanism, to enhance the yield of the ions that we want accelerate and to improve their ion energy and angular emission distributions.

In this paper the influence of the laser parameters, such as the contrast, of the irradiation conditions, as the focal distance from the target surface, and of the target properties, such as the composition and geometry, will be presented, studied and discussed in order to enhance the laser-to-protons and ions energy conversion efficiency and energy distribution.

\section{Materials and methods}

Measurements using $f_{S}$ Ti:sapphire laser have been performed at CELIA-Bordeaux, in France and at IPPLM-Warsaw, in Poland. Further measurements have been performed in PALS-Prague in Czech Republic and useful comparisons of experimental results were performed with literature data by experiments performed in other facilities.

Generally the used lasers are similar, operating at a wavelength of $800 \mathrm{~nm}$, with intensities of the order of $10^{18-19} \mathrm{~W} / \mathrm{cm}^{2}$, with main pulse duration of $30-40 f_{s}$, a pulse energy of about $300 \mathrm{~mJ}$ and a focal spot diameter of 10 microns. However, two different experiments were obtained using a contrast of $10^{5}$ at CELIA Lab and of $10^{9}$ at IPPLM Lab. In the two cases the pedestal duration is about 10 ps.

Experiments were performed using different focal position (FP) distance, which was moved in vacuum with micrometrical step motors, from about -200 microns (in front of the target) up to +200 microns (inside the target). Laser beams used P-polarized radiation with a Gaussian beam distribution.

Many thin targets were used with different composition, multilayers and thickness. Polymers, semiconductors and metals were employed with thicknesses from about 0.1 micron up to about 20 micron. Advanced targets were prepared using thin film of graphene, conductive, transparent to the IR radiation and with high mechanical resistance. Graphene thickness was $10 \mathrm{~nm}$, in order to reduce significantly the prepulse laser reflection effect, increasing the laser-matter energy transfer to the target. Graphene, in facts, has peculiar optical properties, such as high transmittance in the visible and IR regions, above $90 \%$, and very low reflectance, less than $1 \%$ in the visible and near IR regions. It is worthy highlighting graphene's unique optical properties such as its low reflectivity and absorption and high transmission as well as that it produces an unexpectedly high opacity for an atomic monolayer in vacuum, absorbing about $2.3 \%$ of red light [7].

A typical example of reflectance measurements is reported in Fig. 1a for copper and graphene. The plot shows that the copper reflectivity is high, and of the order of $90 \%$ for wavelengths of about $800 \mathrm{~nm}$, while the graphene reflectivity is very low, below $1 \%$, at the used laser wavelengths of $800 \mathrm{~nm}$. Thus, while the direct irradiation of the metallic copper in the first instant of the laser pulse, before all target is vaporized, produces high laser reflections with significant energy loss, the use of a copper surface covered with some graphene monolayers reduces such reflections enhancing the laser energy penetrating the solid target. The trasmittivity of 3 monolayers of grahene, in facts, is high, above $90 \%$, as reported in the plot of Fig. $1 b$.

Thus we expect that the plasma generated by targets with the graphene covering layers should be more energetic than in the case of copper surface directly irradiated in the same experimental conditions.
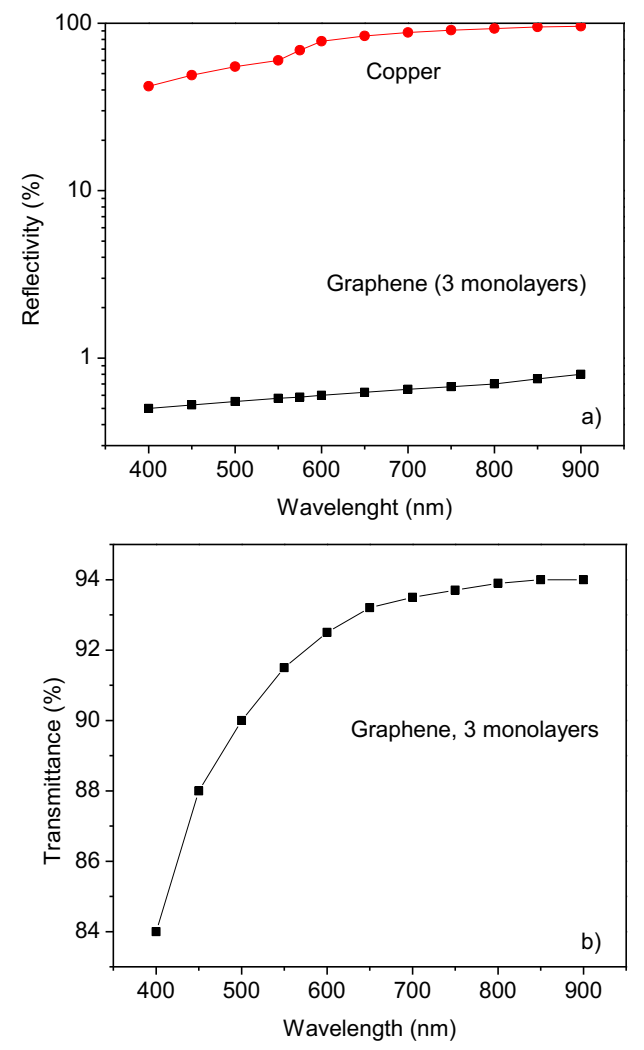

Fig. 1. Reflectivity vs. wavelength for copper and graphene (a) and transmittance vs. wavelength for 3 monolayers of graphene (b).

The plasma diagnostics uses mainly ion collectors (IC), consisting in Faraday cups with the suppressor grid, connected in time-of flight (TOF) configuration with a fast storage oscilloscope and semiconductor detectors, such as $\mathrm{SiC}$ diodes with different active region adapt to detect high energetic ions emitted from plasma using TOF approach [8]. IC and semiconductor detectors are placed at different angles with respect to the normal target surface and at different known distances from the target, in order to detect the forward and the backward ion acceleration.

To use TNSA regime driving targets thinner than $1 \mu \mathrm{m}$ maintaining high contrast it is necessary to

maintain undisturbed the target rear surface, other while only ponderomotive ion acceleration occurs in plasma 
and no so coordinate manner and a lower forward ion acceleration can be recorded.

A typical experimental set-up scheme is reported in Fig. $2 \mathrm{a}$, in which different diagnostics are employed in TOF approach using diamonds, silicon, $\mathrm{SiC}$ and IC detectors. Fig. 2 b shows the typical $f_{s}$ laser pulse and its pedestal relative to the CELIA Lab laser.

\section{Results}

In order to understand the experimental differences obtained irradiating the same target with two lasers having different contrast, a target constituted by graphene/Cu layers, $10 \mathrm{~nm}$ in thickness deposited on a $\mathrm{Cu}$ substrate (14 $\mu \mathrm{m}$ in thickness), was irradiated from the graphene face and the $\mathrm{Cu}$ as rear surface. In both cases the laser focal spot was 10 microns in diameter focused on the target surface $(\mathrm{FP}=0)$.
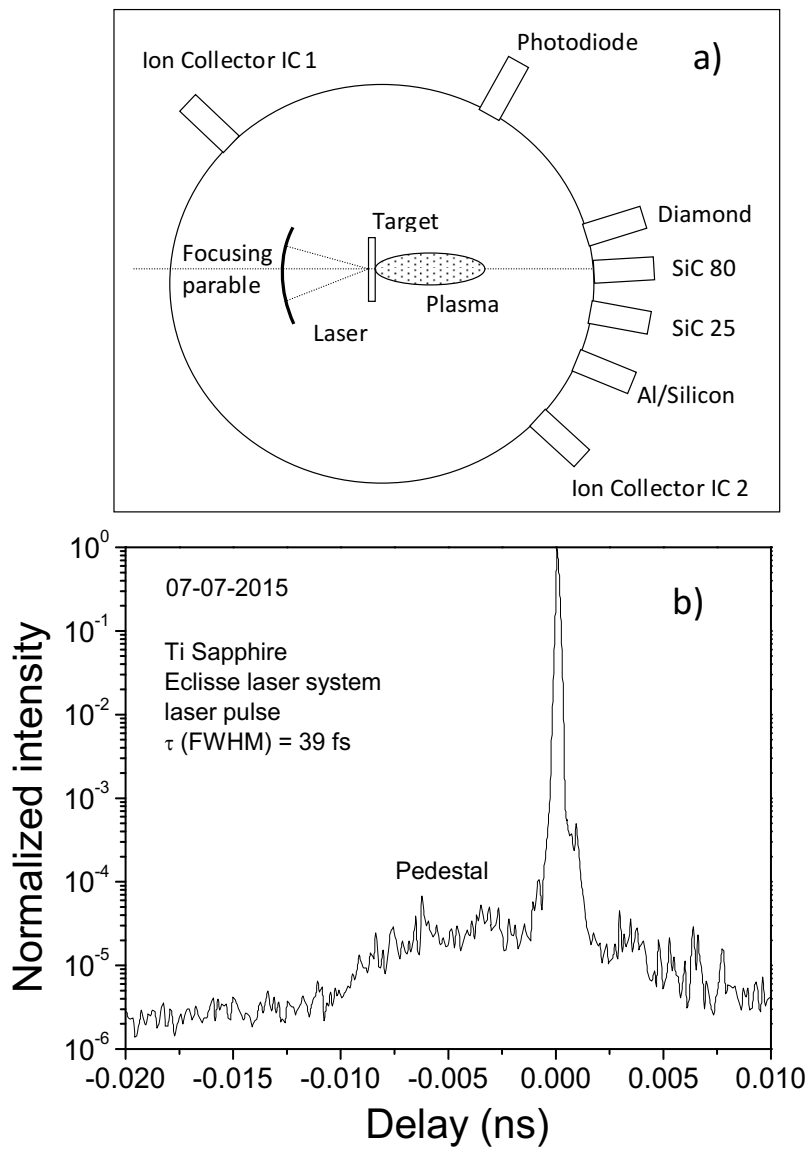

Fig. 2. Scheme of the experimental set-up (a) and pulse shape intensity vs. time of the Eclipse laser in Celia Laboratory (b).

Fig. 3a reports the $\mathrm{SiC}$ spectrum (SiC80) of the plasma forward $\left(0^{\circ}\right)$ radiations detected at a flight distance of $72.5 \mathrm{~cm}$ using a contrast of $10^{5}$, while Fig. $3 \mathrm{~b}$ that of $\mathrm{SiC}$, in forward emission $\left(0^{\circ}\right)$, at a flight distance of 78.6 $\mathrm{cm}$ using a contrast value of $10^{9}$.

The elements expected from the ion acceleration, due to the target composition, are protons (as contamination), carbon and copper, of which these last must be predominant. In fact, the spectra shows such ions with a main peak due to $\mathrm{Cu}$ ions but which energy, calculate from TOF approach, is very different in the two cases.
At low laser contrast (Fig. 3a) the protons are detected at about $92 \mathrm{~ns}$, thus by considering the flight distance of $74.5 \mathrm{~cm}$ their maximum kinetic energy is about $330 \mathrm{keV}$, the carbon are detected at about $2.0 \mathrm{MeV}$ and the copper at about 9.2 MeV. Such measurements are agree with an ion acceleration of about $330 \mathrm{keV}$ per charge state, in fact, assuming the carbon and the copper atoms to be all ionized, the more energetic $\mathrm{C}^{6+}$ and $\mathrm{Cu}^{29+}$ ions should have an energy of $6 \times 330 \mathrm{keV} \sim 2 \mathrm{MeV}$ and $29 \times 330 \mathrm{keV} \sim$ 9.5 MeV, respectively, in good agreement with that detected. Moreover, in this case the maximum spectrum ion yield is low, of about $1.5 \mathrm{mV}$.

At high laser contrast (Fig. 3b) the protons are detected at a maximum kinetic energy of about $600 \mathrm{keV}$, the carbon at about $3.6 \mathrm{MeV}$ and the copper at about 16 $\mathrm{MeV}$. Such measurements are agree with an ion acceleration of about $600 \mathrm{keV}$ per charge state, in fact, assuming the carbon and the copper atoms to be all ionized, the more energetic $\mathrm{C}^{6+}$ and $\mathrm{Cu}^{29+}$ ions should have an energy in agreement with that detected. Moreover, in this case the maximum ion yield of the spectrum is high, of about $2.3 \mathrm{~V}$.
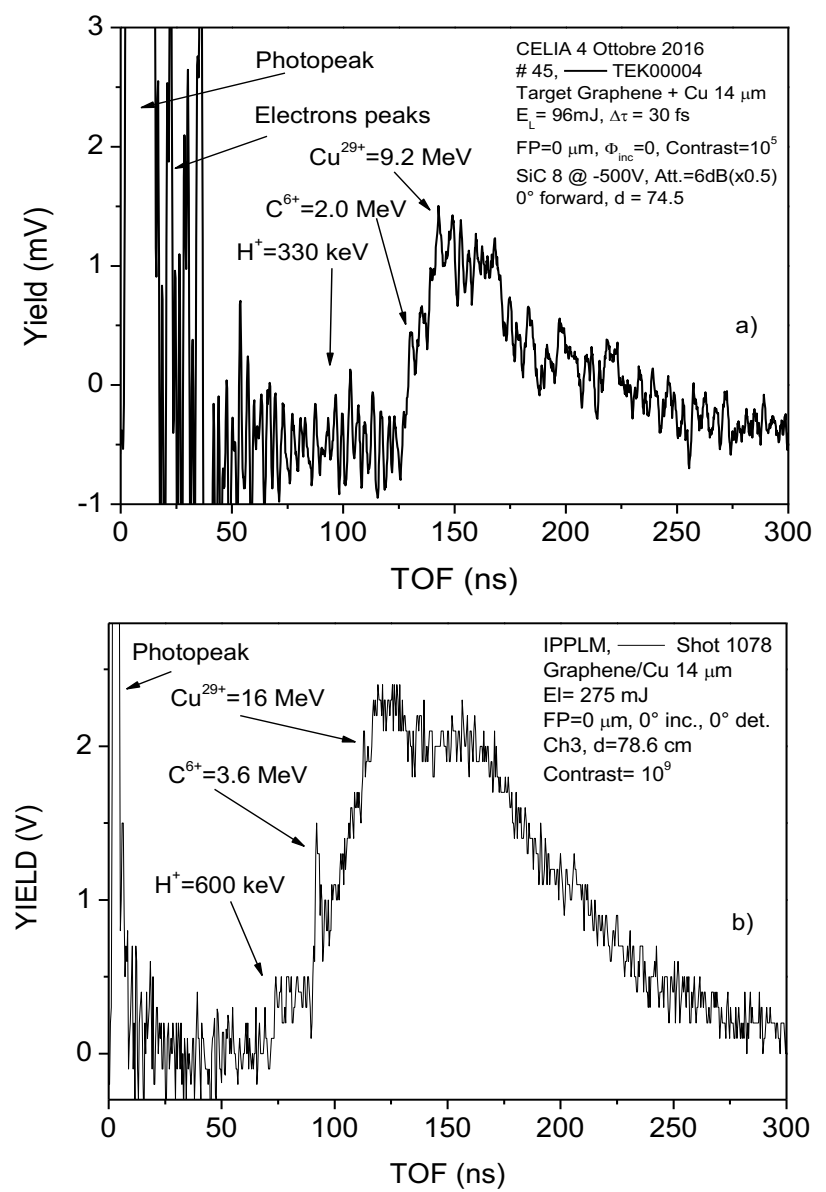

Fig. 3. Typical SiC-TOF spectrum obtained detecting the radiations emitted in forward direction irradiating a graphene/Cu target at low contrast (a) and at high contrast (b).

Thus the higher contrast permits to maintain low the pedestal that limits the ablation of the target ensuring that the rear side of the target remains solid and that TNSA regime may occur. The higher contrast produces higher electron density and temperature and 
consequently, according to eq. (1), higher electric field driving the forward ion acceleration. Not only the ion energy is increased by about a factor two but also the ion yield enhances strongly and of about a factor higher than 1500 , by considering that the same detector in this second case is more distant form the target. The use of different focal positions generates different plasmas due to the different spot diameter that changes the laser intensity and to the possibility to induce preplasma in which not linear self-focusing effects can be produced. The properties of the produced plasma change by irradiating the graphene/copper target, 14 micron in copper thickness, by changing the focal position with respect to the target graphene surface.

Fig. $4 \mathrm{a}$ and $4 \mathrm{~b}$ report two typical $\mathrm{SiC}$ spectra ( $\mathrm{SiC} 80$ ) obtained using two different focal positions of $\mathrm{FP}=-100$ microns (in front of the target) and $\mathrm{FP}=+100$ micron (inside the target), respectively. In the first case the maximum ion energy is measured at $275 \mathrm{keV}, 1.75 \mathrm{MeV}$ and 7.6 MeV for protons, carbon ions and copper ions, respectively, indicating a mean charge acceleration of about $275 \mathrm{keV}$ per charge state. In the second case the maximum ion energy is measured at $1.6 \mathrm{MeV}, 9.7 \mathrm{MeV}$ and $40 \mathrm{MeV}$ for protons, carbon ions and copper ions, respectively, indicating a mean charge acceleration of about $1.6 \mathrm{MeV}$ per charge state.
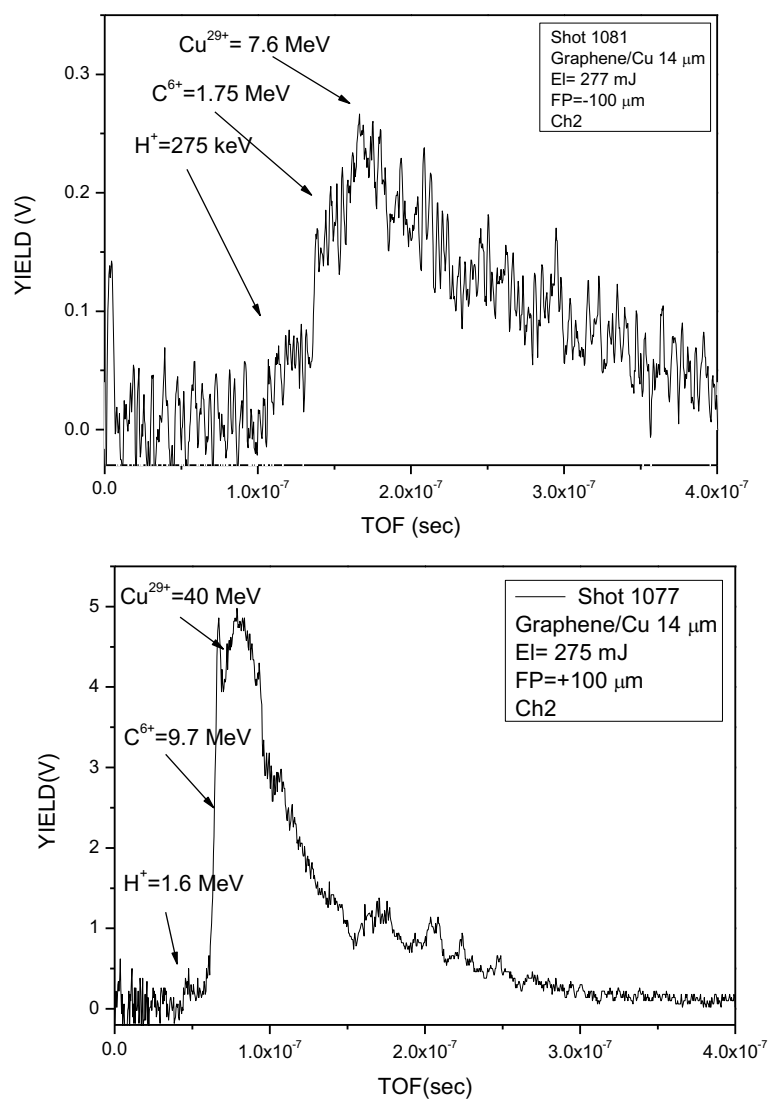

Fig. 4. Typical SiC-TOF spectrum obtained irradiating the graphene/Cu foil with high contrast laser with a focal position of -100 microns (in front of the target surface) (a) and +100 microns (inside the target surface) (b).

This result is particularly interesting because the only focal shift of 200 microns determines an ion energy increase of about a factor 5.8, demonstrating that the focalization on the copper foil inside the target, instead that on the superficial graphene layers, increases the relativistic electron emission from the target and consequently the forward electric field driving the ion acceleration.

The plots of Fig. 5a and $5 \mathrm{~b}$ report the results about the proton accelerations obtained using a laser contrast of $10^{5}$ and $10^{9}$, respectively, irradiating the same target of graphene/copper at different focal positions. The fist plot demonstrates that the maximum proton acceleration is obtained at $\mathrm{FP}=0$ microns, decreasing at the other focal positions, while the second plot demonstrated that the maximum proton acceleration is obtained focusing at $\mathrm{FP}=+100$ microns, confirming a different process occurring in the target. In this second case, thank to the high laser contrast, the laser pedestal don't destroy the graphene superficial layer that transmits the laser pulse and hits the copper surface producing TNSA ion acceleration from the rear face of the copper. In the first case, instead, due to the low laser contrast the pedestal destroys the graphene layer and part of the copper surface, lower laser energy accelerates electrons and TNSA ion acceleration occurs also but with a less ion acceleration process.
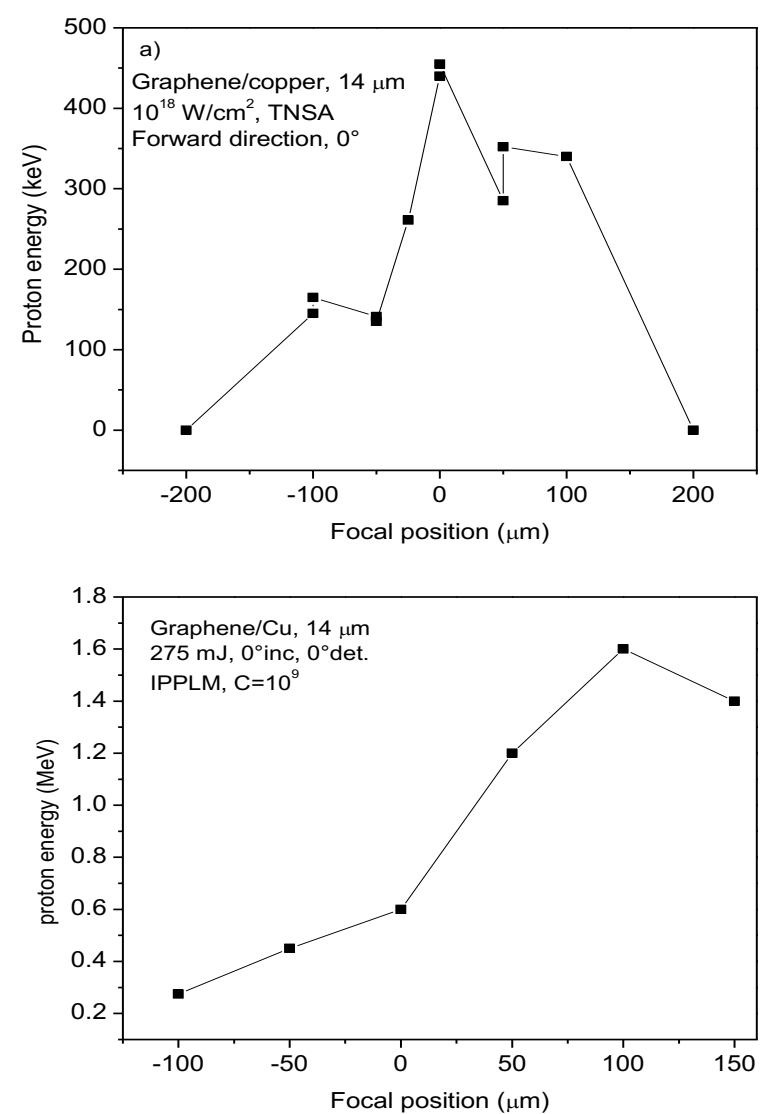

Fig. 5. Measured proton energy as a function of the focal position for $f_{s}$ laser at low contrast (a) and high contrast (b).

The maximum ion acceleration exhibits a clear trend with the target thickness influencing the accelerated electron transmission from the thin foil in term of quantity, energy and angular spreading. The number of accelerated protons and the proton energy changes with the target composition and thickness. According to eq. 
(1), by increasing the rear hot electron temperatures and densities enabling the higher protons and ions acceleration. Generally using thinner targets more energetic electrons are transmitted from the target surface to its rear side and the electric field driving the Generally the proton acceleration is too low if the target is thick or is very thin, and assumes a maximum value for an optimal thickness that maximize the electron transmission to the rear side of the foil. The accelerated surface electrons, their energy loss and transversal spreading inside the target modify the ion acceleration and permit to generate a maximum electrical field if their emission has spatial and temporal coherence form the rear face of the target. The proton yield instead depends mainly on the target composition, being high for hydrogenate targets, such as polymers and hydrates, and low for hydrogen contamination of surfaces.

At high laser contrast, for low target thickness, the maximum proton energy increases because more electrons are injected in the rear surface and a better charge separation occurs increasing the forward electric field driving the ion acceleration. Thus, in such conditions it is possible to obtain high proton energies for submicrometric target thicknesses.
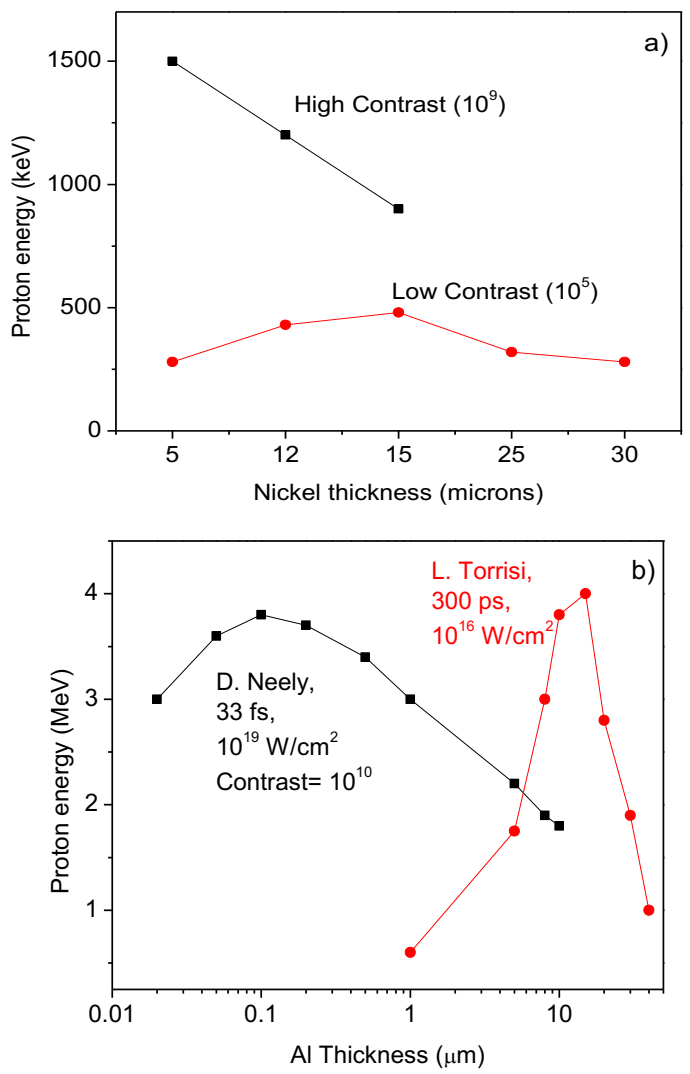

Fig. 6. Measured proton energy vs. target thickness for Ni (a) and $\mathrm{Al}$ (b) thin films as a function of the pulse contrast and of the pulse duration, respectively.

Of course too low foil thickness reduce the number of particles to negligible values and determine a very low flux of relativistic electrons up to a reduced charge separation and a limit in the ion acceleration is obtained. As reported in literature [9] sometimes at high laser contrast, of the order of $10^{10}$ the proton energy continues to increase also up to sub-micrometric thicknesses, possibly due to an increasing role of electron recirculation with decreasing the target thickness. Below about $100 \mathrm{~nm}$, we find a decrease in the maximum proton acceleration, resulting in a clear optimum in thickness.

This decrease could be due to the finite temporal contrast on the picosecond time scale, given by the shoulders of the main pulse rather than to ASE or separate prepulses.

Fig. $6 b$ reports the result of the dependence on the protons energy accelerated from a thin $\mathrm{Al}$ target irradiated at $10^{16} \mathrm{~W} / \mathrm{cm}^{2}$ using the PALS iodine laser operating at $1315 \mathrm{~nm}$ wavelength and $300 \mathrm{ps}$, described by L. Torrisi [10] reaching about $4 \mathrm{MeV}$ energy at the optimum target thickness of 10 microns Al in TNSA regime. For comparison the same plot shows the results obtained by D. Neely [9] using the LLC laser which delivers $33 \mathrm{fs}$ pulses with a $1 \mathrm{~ns}$ duration ASE, at an intensity contrast of $2 \times 10^{8}$ and a contrast of $10^{10}$, when it is focused to an intensity of $10^{19} \mathrm{~W} / \mathrm{cm}^{2}$ on $\mathrm{Al}$ foils. In this case the optimal thickness to reach the maximum proton energy of about $4 \mathrm{MeV}$ is obtained at 0.1 microns. The comparison shows that the different laser intensity and temporal pulses optimize the proton acceleration in two different thickness regions, of the order of tens microns in the first case and of $100 \mathrm{~nm}$ in the second case, respectively.

The laser conversion efficiency in proton acceleration can be calculated accurately knowing the ion current, the ion energy distributions and the angular emission distribution from the target. The $\mathrm{SiC}$ detector spectra, showing the ion current versus time, permits to give an evaluation of the conversion efficiency taking in consideration the average current of the diode, $I=V / R$. For example from the spectra of Fig. $3 b$ referred to high contrast, it is possible to evaluate the real proton current by the equation:

$$
I_{p}=\frac{V}{R} \cdot \frac{E_{c}}{E_{p}}=\frac{0.4 \mathrm{~V}}{50 \Omega} \cdot \frac{7.78 \mathrm{eV}}{3.10^{5} \mathrm{eV}}=0.21 \mu \mathrm{A}
$$

where $V$ is the ion yield in Volt, $\mathrm{R}$ is the input resistance of the storage oscilloscope of $50 \Omega, \mathrm{E}_{\text {c }}$ represents the energy to produce a pair in the semiconductor $(7.78 \mathrm{eV})$ and $\mathrm{E}_{\mathrm{p}}$ is the mean energy of the protons, supposed to be $300 \mathrm{keV}$.

The number $\mathrm{N}$ of protons produced by a single laser shot can be calculated as follows:

$$
\begin{aligned}
& N=I_{p} \frac{\Delta t}{e} \cdot \frac{\Omega}{\Delta \Omega}= \\
& =0.21 \mu A \cdot \frac{25 n s}{1.6 \cdot 10^{-19} C} \cdot \frac{1.75 s r}{6.5 \mu s r}=8.8 \cdot 10^{9}
\end{aligned}
$$

where $\Delta t$ is the proton current duration, $e$ is the electron charge, $\Omega$ is the total solid angle of proton emission, assumed of $1.75 \mathrm{sr}$, and $\Delta \Omega$ is the solid angle of detection $(6.5 \mu \mathrm{sr})$. 
Assuming these ions have a mean kinetic energy $\left(\mathrm{E}_{\max }\right)$ of $300 \mathrm{keV}$ and the laser pulse energy to be $275 \mathrm{~mJ}\left(\mathrm{E}_{\mathrm{L}}\right)$, thus the laser conversion efficiency in protons acceleration is:

$$
\begin{aligned}
& \eta(\%)=\frac{N \cdot E_{\max }}{E_{L}} \cdot 100= \\
& \frac{8.8 \cdot 10^{9} \cdot 3 \cdot 10^{5} \cdot 1.6 \cdot 10^{-19} \mathrm{~J}}{275 \cdot 10^{-3} \mathrm{~J}} \cdot 100=0.15 \%
\end{aligned}
$$

Of course the conversion efficiency of proton acceleration using low laser contrast will be lower, as immediately observable from their yield of about a factor 1500 lower and minor current and energy.

\section{Discussion and Conclusions}

The process of TNSA ion acceleration depends on many parameters connected to the used laser system, to the irradiation conditions and to the target properties.

The laser contrast is fundamental because if it is low it means that the pedestal pulse contains high laser energy which will hit the target destroying it totally or partially, depending on its thickness and composition, before that the main pulse arrives after tens $p s$. To use TNSA acceleration mechanism the rear side of the target must be maintained solid during the main pulse arrive and the contrast must be more high possible.

The irradiation conditions are also essential to be controlled accurately, as demonstrated, for instance, from the different laser focal position with respect to the target surface depend the ion acceleration. The optimal acceleration condition must be searched changing the focal position and maximizing the proton energy.

The target properties are also very important because from its composition depends the main ion stream to be accelerated, from its thickness depends the maximum ion acceleration and from its properties depends the maximum laser energy transferred to the target. For example the use of thin graphene layers reduces the reflectivity maintaining high its transmission, permitting high energy transfer to the substrate on which the film is deposited. Its use is recommended when high laser contrast is employed.

Although many factors are under control, high intensity laser are used and advanced target permit to transfer high energy from photons to the matter, the ion beam conversion energy remains low, as reported in many literature $[11,12]$.

A laser-to-proton beam energy conversion efficiency of $0.1 \%$, for protons with maximum energy of $600 \mathrm{keV}$ is obtained from our calculations. This efficiency is based on the use of advanced targets with absorbent graphene superficial layers and very short pulses from a Ti:sapphire laser operating at about $0.3 \mathrm{~J}$. With a contrast of $10^{9}$, the optimum target thickness is found to be of about 5 microns or lower, in agreement with literature reporting the optimal thickness at $10^{10}$ contrast of about 0.1 micron. The results show a significant enhancement in proton beam scaling with a variation in target thickness and clearly demonstrate the benefits of using an ultrahigh contrast laser to drive thin targets.

This work was supported by the "Research and Mobility" project of Messina University No. 74893496, scientifically coordinated by Professor L. Torrisi. It has been implemented at the CANAM (Center of Accelerators and Nuclear Analytical Methods) infrastructure LM2015056 and has been supported by project No. P108/12/G108.

\section{References}

1. L. Torrisi, Physics of Plasma 24, 023111 (2017)

2. R. Prasad, S.Ter-Avetisyan, D. Doria, K.E. Quinn, L. Romagnani, P.S. Foster, C.M. Brenner, J.S. Green, P. Gallegos, M.J.V. Streeter, D.C. Carroll, O. Tresca, N.P. Dover, C.A.J. Palmer, J. Schreiber, D. Neely, Z. Najmudin, P. McKenna, M. Zepf, M. Borghesi, Nucl. Instr. and Methods in Physics Res. A 653, 113 (2011).

3. L. Torrisi, M. Cutroneo, and J. Ullschmied, Physics of Plasmas 24, 043112 (2017).

4. S. Eliezer (Ed). The interaction of high power lasers with plasmas. Bristol: Institute of Physics Publishing, (2012).

5. I.O. Musgrave, C. Hernandez-Gomez, D. Canny, R. Heathcote, R.J. Clarke, J.L. Collier, S. Bandyopadhyay, Central Laser Facility Annual Report, 197 (2004/2005).

6. L. Laska, K. Jungwirth, J. Krasa, E. Krousky, M. Pfeifer, K. Rohlena, J. Ullschmied, J. Badziak, P. Parys, J. Wolowski, S. Gammino, L. Torrisi, F.P. Boody Laser and Particle Beams 24(1), 175 (2006).

7. M. Aliofkhazraei, N. Ali, W.I. Milne, C.S. Ozkan, S. Mitura, J.L. Gervasoni, Graphene Science Handbook, Electrical and Optical Properties CRC Press , Taylor \& Francis, London (2016).

8. M. Cutroneo, P. Musumeci, M. Zimbone, L. Torrisi, F. La Via, D. Margarone, A. Velyhan, J. Ullschmied, L.Calcagno, J. Mat. Research 28(01), 87 (2012).

9. D. Neely, P. Foster, A. Robinson, F. Lindau, O. Lundh, A. Persson, C.G. Wahlström, P. McKenna, Applied Physics Letters 89, 021502 (2006).

10. L. Torrisi and M. Cutroneo, Journal of Applied Physics 115, 083105 (2014).

11. S. Kar, K.F. Kakolee, B. Qiao, A. Macchi, M. Cerchez, D. Doria, M. Geissler, P. McKenna, D. Neely, J. Osterholz, R. Prasad, K. Quinn, B. Ramakrishna, G. Sarri, O. Willi, X.Y Yuan, M. Zepf, M. Borghesi, Phys. Rev. Lett. 109, 185006 (2012).

12. M. Roth, A. Blazevic, M. Geissel, T. Schlegel, T.E. Cowan, M. Allen, J.C. Gauthier, P. Audebert, J. Fuchs, J. Meyer-ter-Vehn, M. Hegelich, S. Karsch, A. Pukhov, Phys. Rev. Spec. Topics 5, 061301 (2002). 